\title{
Heavy-Meson Decay Constants: QCD Sum-Rule Glance at Isospin Breaking
}

\author{
Wolfgang Lucha ${ }^{1, \star}$, Dmitri Melikhov ${ }^{1,2,3, \star \star}$, and Silvano Simula ${ }^{4, \star \star \star}$ \\ ${ }^{1}$ Institute for High Energy Physics, Austrian Academy of Sciences, Nikolsdorfergasse 18, A-1050 Vienna, \\ Austria \\ ${ }^{2}$ D. V. Skobeltsyn Institute of Nuclear Physics, M. V. Lomonosov Moscow State University, 119991, Moscow, \\ Russia \\ ${ }^{3}$ Faculty of Physics, University of Vienna, Boltzmanngasse 5, A-1090 Vienna, Austria \\ ${ }^{4}$ INFN, Sezione di Roma Tre, Via della Vasca Navale 84, I-00146 Roma, Italy
}

\begin{abstract}
QCD sum rules for decay constants of heavy mesons with $u$ or $d$ quark yield for $B$ mesons much less isospin breaking than lattice QCD but good agreement for $D$ mesons.
\end{abstract}

\section{Hadronic properties scrutinized from the point of view of QCD sum rules}

QCD sum rules [1] are analytic relations between hadron features and QCD parameters emerging upon evaluation of correlation functions of appropriate interpolating operators at both the hadronic level and the QCD level by insertion of complete sets of hadron states, exploitation of Wilson's operator product expansion, Borel transformation, and the quark-hadron duality assumption above effective thresholds.

In order to both raise the accuracy of QCD sum-rule predictions and estimate the systematic errors [2], some time ago we developed [3] a modified formalism which takes into account the dependence of the effective thresholds on the Borel parameters. This enabled the improved extraction of heavy-meson decay constants $f$ [4], notably of the inequalities $f_{B_{(s)}^{*}}<f_{B_{(s)}}$ [5], later on confirmed by lattice QCD [6].

QCD sum rules provide in analytic form the dependences of the properties of the hadrons currently under study on the basic parameters of QCD. This allows us to study the impact of the isospin breaking provoked by the slight difference in the masses of the light $u$ and $d$ quarks on the decay constants of the heavy-light mesons, by working out the explicit relation of the decay constants to the light quark mass.

\section{Heavy-meson decay constants: extraction from QCD sum-rule approach}

Hence, let us try to get hold of the dependence of our QCD sum-rule approach on the light-quark mass. We analyse pseudoscalar $\left(P_{q}\right)$ and vector $\left(V_{q}\right)$ mesons (generically labelled $M_{q} \equiv P_{q}, V_{q}$ ) of mass $M_{M_{q}}$, composed of a heavy quark $Q=b, c$ and a light quark $q=u, d$ with masses $m_{Q}$ and $m_{q}$, respectively. In terms of suitable interpolating heavy-light quark-current operators, that is, the axial-vector (A) current $A_{\mu}(x) \equiv \bar{q}(x) \gamma_{\mu} \gamma_{5} Q(x)$ and the vector $(\mathrm{V})$ current $V_{\mu}(x) \equiv \bar{q}(x) \gamma_{\mu} Q(x)$, the decay constants $f_{M_{q}}$ of the mesons [with momentum $p$ and, in the case of vector mesons, polarization vector $\varepsilon_{\mu}(p)$ ] are defined by

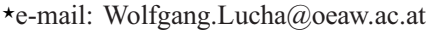

$\star \star$ e-mail: dmitri_melikhov@gmx.de

$\star \star \star$ e-mail: simula@roma3.infn.it
} 


$$
\left\langle 0\left|A_{\mu}(0)\right| P_{q}(p)\right\rangle=\mathrm{i} f_{P_{q}} p_{\mu}, \quad\left\langle 0\left|V_{\mu}(0)\right| V_{q}(p)\right\rangle=f_{V_{q}} M_{V_{q}} \varepsilon_{\mu}(p) .
$$

The decay constants may be inferred from the correlators of two of such currents $J_{\mu}(x)=A_{\mu}(x), V_{\mu}(x)$ :

$$
\Pi_{\mu \nu}^{(J)}(p)=\mathrm{i} \int \mathrm{d}^{4} x \exp (\mathrm{i} p x)\left\langle 0\left|T\left(J_{\mu}(x) J_{\nu}^{\dagger}(0)\right)\right| 0\right\rangle, \quad J=\mathrm{A}, \mathrm{V} .
$$

The QCD sum rules providing, for the mesons $M_{q}=P_{q}, V_{q}$, their masses $M_{M_{q}}$ and decay constants $f_{M_{q}}$ receive both purely perturbative contributions, represented by dispersion integrals of spectral densities $\rho_{J}\left(s, m_{Q}, m_{q}, \alpha_{\mathrm{s}}\right)$, given by series expansions in powers of the strong coupling $\alpha_{\mathrm{s}}$, and non-perturbative contributions $\widehat{\Pi}_{N}^{(J)}\left(\tau, m_{Q}, m_{q}\right)$, parametrized by vacuum condensates; generically, they assume the form $f_{M_{q}}^{2} M_{M_{q}}^{2 N} \exp \left(-M_{M_{q}}^{2} \tau\right)=\int_{\left(m_{Q}+m_{q}\right)^{2}}^{s_{\mathrm{eff}}(\tau)} \mathrm{d} s s^{N} \exp (-s \tau) \rho_{J}\left(s, m_{Q}, m_{q}, \alpha_{\mathrm{s}}\right)+\widehat{\Pi}_{N}^{(J)}\left(\tau, m_{Q}, m_{q}\right), \quad J=\mathrm{A}, \mathrm{V}$.

The integer $N$ relates to the Lorentz nature of the employed interpolating operator: the axial-vector and vector currents $A_{\mu}(x)$ and $V_{\mu}(x)$ imply $N=1$, whereas, for pseudoscalar currents, we would get $N=2$.

The innovative change introduced, in Refs. [3], to the concept of QCD sum rules simply consists in realizing (and accepting) that, in general, the effective threshold $s_{\text {eff }}$ will depend on the Borel variable $\tau$ (the parameter of dimension of inverse mass squared arising upon Borel transformation): $s_{\text {eff }}=s_{\text {eff }}(\tau)$. Our favourite possibility for acquiring information on the actual $\tau$ behaviour of $s_{\text {eff }}(\tau)$ is to fit our QCD sum-rule predictions of the masses of the mesons under study to their experimentally measured values. It suffices to model $s_{\text {eff }}(\tau)$ by polynomial ansätze of rather low order $n$, with expansion coefficients $s_{j}^{(n)}$ :

$$
s_{\mathrm{eff}}^{(n)}(\tau)=\sum_{j=0}^{n} s_{j}^{(n)} \tau^{j}
$$

By its central value and half-width, the spread of results for linear $(n=1)$, quadratic $(n=2)$, and cubic $(n=3) s_{\text {eff }}^{(n)}(\tau)$ behaviour enables an estimate of some decay constant and its systematic uncertainty [3].

We should achieve our goals by just imitating the application of our algorithm [3] to the analysis of heavy-meson decay constants in related studies [4, 5] based on cutting-edge QCD contributions [7-9]. In order to optimize our predictions' perturbative performance [8], we use quark masses defined by the modified minimal-subtraction $(\overline{\mathrm{MS}})$ renormalization scheme. Table 1 shows all numerical ingredients.

Table 1. Numerical parameter values entering in QCD sum rules for heavy-meson decay constants $[4,8,10,11]$.

\begin{tabular}{lcc}
\hline \multicolumn{2}{c}{ Operator-product-expansion parameter } & Numerical input value \\
\hline$u$-quark $\overline{\mathrm{MS}}$ mass & $m_{u}(2 \mathrm{GeV})$ & $\left(2.3_{-0.5}^{+0.7}\right) \mathrm{MeV}$ \\
$d$-quark $\overline{\mathrm{MS}}$ mass & $m_{d}(2 \mathrm{GeV})$ & $\left(4.8_{-0.3}^{+0.5}\right) \mathrm{MeV}$ \\
$s$-quark $\overline{\mathrm{MS}}$ mass & $m_{s}(2 \mathrm{GeV})$ & $(93.8 \pm 2.4) \mathrm{MeV}$ \\
$c$-quark $\overline{\mathrm{MS}}$ mass & $m_{c}\left(m_{c}\right)$ & $(1275 \pm 25) \mathrm{MeV}$ \\
$b$-quark $\overline{\mathrm{MS}}$ mass & $m_{b}\left(m_{b}\right)$ & $(4247 \pm 34) \mathrm{MeV}$ \\
Strong coupling & $\alpha_{\mathrm{s}}\left(M_{Z}\right)$ & $0.1185 \pm 0.0006$ \\
Light-quark condensate & $\langle\bar{q} q\rangle(2 \mathrm{GeV})$ & $-[(267 \pm 17) \mathrm{MeV}]^{3}$ \\
Strange-quark condensate & $\langle\bar{s} s\rangle(2 \mathrm{GeV})$ & $(0.8 \pm 0.3) \times\langle\bar{q} q\rangle\left(2 \mathrm{GeV}^{2}\right)$ \\
Gluon condensate & $\left\langle\frac{\alpha_{\mathrm{s}}}{\pi} G \mathrm{GG}\right.$ & $(0.024 \pm 0.012) \mathrm{GeV}^{4}$ \\
\hline
\end{tabular}




\section{Summary of procedures, results, insights, conclusions, and outlook [12]}

Allowing the light-quark mass $m_{q}$ to assume any value within the interval $0 \leq m_{q} \leq m_{s}$ and defining on this domain a function $f_{M}\left(m_{q}\right)$ by the outcomes of our QCD sum rule for the decay constant of a meson $M$ with light quark of just this mass $m_{q}$, we minimize uncertainties by analyzing the difference quotient

$$
\frac{f_{M}\left(m_{d}\right)-f_{M}\left(m_{u}\right)}{m_{d}-m_{u}},\left.\quad M\right|_{m_{q}=m_{u}, m_{d}, m_{s}}=M_{u, d, s},
$$

or the slope of $f_{M}\left(m_{q}\right)$ in the vicinity of $\bar{m} \equiv\left(m_{u}+m_{d}\right) / 2$, requiring us to specify some $m_{q}$ dependences:

- For the light-quark condensate, $\langle\bar{q} q\rangle$, we assume a linear $m_{q}$ dependence from its value $\langle\bar{u} u\rangle \approx\langle\bar{d} d\rangle$ at $\bar{m} \equiv\left(m_{u}+m_{d}\right) / 2=3.5_{-0.2}^{+0.7} \mathrm{MeV}[10]$ up to the strange-quark condensate, $\langle\bar{s} s\rangle$, at $m_{s}=93.8 \mathrm{MeV}$.

- For the mass $M_{M}\left(m_{q}\right)$ of any heavy-light $[\bar{Q} q]$ meson, we allow for a linear $m_{q}$ dependence from the experimental [10] nonstrange-meson mass $M_{M_{u d}}$ up to the associated strange-meson mass $M_{M_{s}}$ [10]:

$$
M_{M}\left(m_{q}\right)=M_{M_{u d}}+\frac{m_{q}-\bar{m}}{m_{s}-\bar{m}}\left(M_{M_{s}}-M_{M_{u d}}\right) .
$$

Figure 1 shows the decay-constant function $f_{M}\left(m_{q}\right)$ for all $M=B^{(*)}, D^{(*)}$, whereas Fig. 2 illustrates nicely the reduction of the systematic errors, when normalizing $f_{M}\left(m_{q}\right)$ to their values $f_{M}(0)$ at $m_{q}=0$.

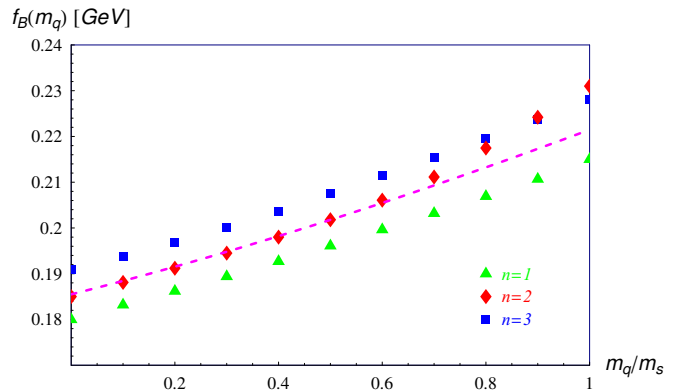

(a)

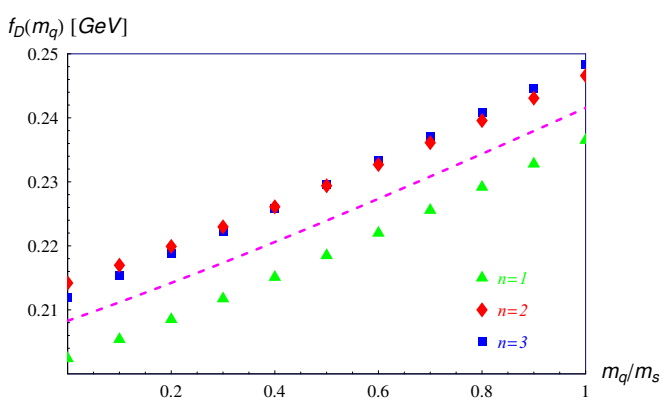

(c)

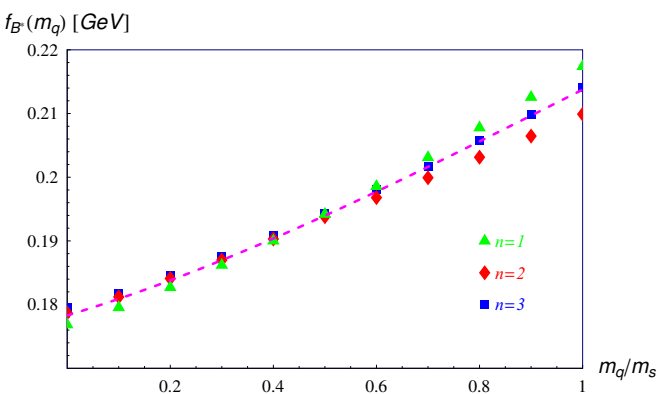

(b)

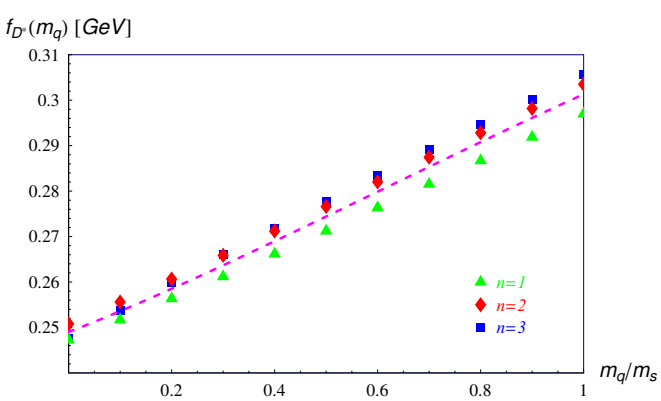

(d)

Figure 1. Decay constants $f_{M}$ of both $[\bar{b} q]$ beauty mesons $B$ (a) and $B^{*}(\mathrm{~b})$ and $[\bar{c} q]$ charmed mesons $D(\mathrm{c})$ and $D^{*}$ (d): dependence on the light quark mass $m_{q}$ predicted by QCD sum rules [3] employing, for the effective threshold $s_{\mathrm{eff}}(\tau)$, polynomial ansatzes of order $n=1$ (green triangles $\Delta$ ), $n=2$ (red diamonds $\downarrow$ ), and $n=3$ (blue squares $\mathbf{\square}$ ). The dashed magenta line indicates the position of the center of the band spanned by these individual- $n$ extractions. 


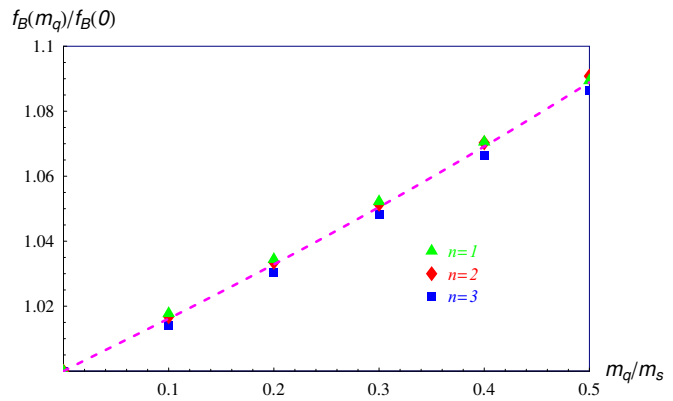

(a)

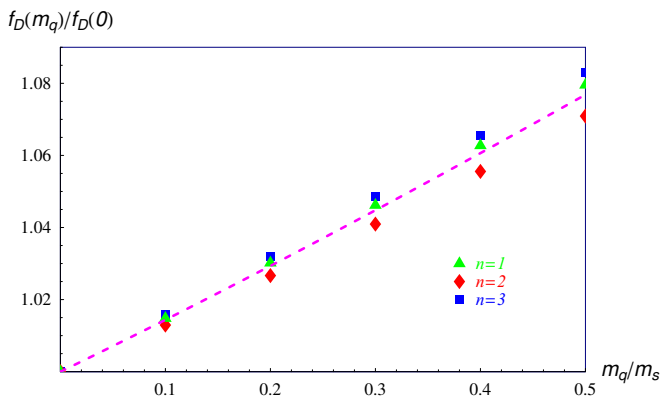

(c)

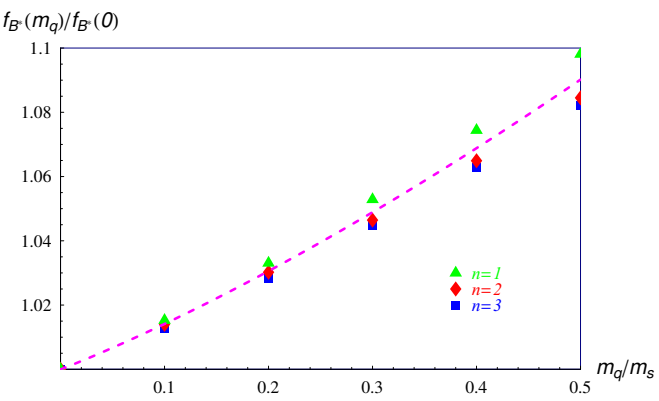

(b)

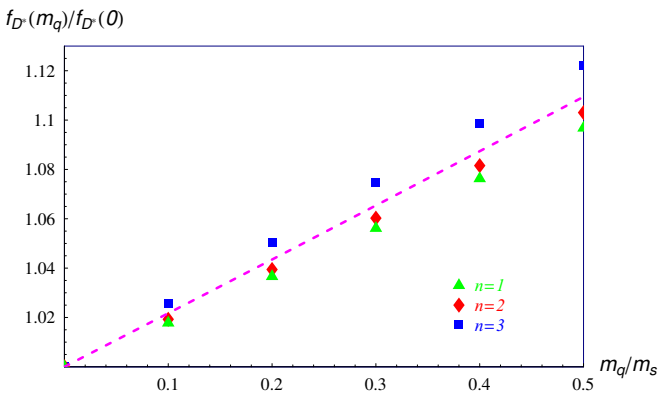

(d)

Figure 2. Decay-constant ratios, $f_{M}\left(m_{q}\right) / f_{M}(0)$, of the bottom mesons $B$ (a) and $B^{*}($ b) and the charmed mesons $D$ (c) and $D^{*}(\mathrm{~d})$ : dependence on the light-quark mass $m_{q}$ from QCD sum rules [3] relying, for the effective threshold $s_{\text {eff }}(\tau)$, on polynomial ansätze of order $n=1$ (green triangles $\Delta$ ), $n=2$ (red diamonds $\downarrow$ ) or $n=3$ (blue squares $\mathbf{\square}$ ). The dashed magenta line indicates the position of the center of the band spanned by these individual $n$ extractions.

Albeit, as physical quantities, the decay constants $f_{M_{q}}$ must not depend on the renormalization scale $\mu$, inevitable truncations of perturbative expansions induce artificial $\mu$ dependences of any QCD sum-rule results, raising the errors. We find $f_{M_{q}}$ from the ranges $[4,5] 1<\mu(\mathrm{GeV})<3$ for charmed mesons and $3<\mu(\mathrm{GeV})<5$ for beauty mesons. For each of the mesons analyzed, $f_{M}\left(m_{q}\right)$ may be parametrized as

$$
\frac{f_{M}\left(m_{q}\right)}{f_{M}(0)}=1+r_{\ell} \frac{m_{q}}{m_{s}} \log \frac{m_{q}}{m_{s}}+r_{1} \frac{m_{q}}{m_{s}}+\cdots, \quad M=B^{(*)}, D^{(*)}
$$

Table 2. Difference of the decay constants $f_{M_{q}}$ for $d$-vs. $u$-quark mesons signalling isospin breaking, normalized to $f_{M}(0)$, for $M=B^{(*)}, D^{(*)}$, and numerical values of the coefficients $r_{\ell, 1}$ in the parametrization (2) of these ratios.

\begin{tabular}{lclc}
\hline Meson $M_{q}$ & \multicolumn{1}{c}{$r_{\ell}$} & \multicolumn{1}{c}{$r_{1}$} & $\frac{f_{M_{d}}-f_{M_{u}}}{f_{M}(0)}$ \\
\hline$B$ & $0.011 \pm 0.008$ & $0.181 \pm 0.003$ & $(4.1 \pm 0.4) \times 10^{-3}$ \\
$B^{*}$ & $0.024 \pm 0.003$ & $0.19 \pm 0.02$ & $(3.6 \pm 0.3) \times 10^{-3}$ \\
$D$ & $0.006 \pm 0.015$ & $0.156 \pm 0.010$ & $(3.8 \pm 0.4) \times 10^{-3}$ \\
$D^{*}$ & $0.002 \pm 0.010$ & $0.22 \pm 0.010$ & $(5.7 \pm 1.2) \times 10^{-3}$ \\
\hline
\end{tabular}


Table 2 reveals, for each meson $M_{q}$, the values of the coefficients $r_{\ell, 1}$ and the decay-constant difference $f_{M_{d}}-f_{M_{u}}$, which satisfies $\left(f_{M_{d}}-f_{M_{u}}\right) /\left(m_{d}-m_{u}\right)>0$, tantamount to $f_{M_{d}}>f_{M_{u}}$ for all $M=B^{(*)}, D^{(*)}[12]$. Upon taking into account all the uncertainties induced by the QCD parameters and the systematic ones inherent to the QCD sum-rule technique, we arrive at the following $f_{B^{(*)}, D^{(*)}}$ decay-constant differences:

$$
\begin{array}{ll}
f_{B^{0}}-f_{B^{ \pm}}=(0.79 \pm 0.14) \mathrm{MeV}, & f_{B^{* 0}}-f_{B^{* \pm}}=(0.65 \pm 0.10) \mathrm{MeV}, \\
f_{D^{ \pm}}-f_{D^{0}}=(0.78 \pm 0.13) \mathrm{MeV}, & f_{D^{* \pm}}-f_{D^{* 0}}=(1.41 \pm 0.42) \mathrm{MeV} .
\end{array}
$$

Comparing with related lattice-QCD outcomes [13], our predictions for the decay-constant differences are, for the $B$ mesons, lower than their lattice-QCD counterparts but agree, in the case of the $D$ mesons, with lattice-QCD results. Our findings are of similar size as those got by lattice QCD for the $K$ mesons. In Ref. [12], we present a more detailed analysis, including the uncertainties of the light-quark masses.

Acknowledgement. D. M. was supported by the Austrian Science Fund (FWF): Project P29028-N27.

\section{References}

[1] M. A. Shifman, A. I. Vainshtein, and V. I. Zakharov, Nucl. Phys. B 147 (1979) 385.

[2] W. Lucha, D. Melikhov, and S. Simula, Phys. Rev. D 76 (2007) 036002, arXiv:0705.0470 [hepph]; Phys. Lett. B 657 (2007) 148, arXiv:0709.1584 [hep-ph]; Phys. At. Nucl. 71 (2008) 1461; Phys. Lett. B 671 (2009) 445, arXiv:0810.1920 [hep-ph]; D. Melikhov, Phys. Lett. B 671 (2009) 450, arXiv:0810.4497 [hep-ph].

[3] W. Lucha, D. Melikhov, and S. Simula, Phys. Rev. D 79 (2009) 096011, arXiv:0902.4202 [hepph]; J. Phys. G 37 (2010) 035003, arXiv:0905.0963 [hep-ph]; Phys. Lett. B 687 (2010) 48, arXiv: 0912.5017 [hep-ph]; Phys. At. Nucl. 73 (2010) 1770, arXiv:1003.1463 [hep-ph]; W. Lucha, D. Melikhov, H. Sazdjian, \& S. Simula, Phys. Rev. D 80 (2009) 114028, arXiv:0910.3164 [hep-ph].

[4] W. Lucha, D. Melikhov, and S. Simula, J. Phys. G 38 (2011) 105002, arXiv:1008.2698 [hep-ph]; Phys. Lett. B 701 (2011) 82, arXiv:1101.5986 [hep-ph]; Phys. Rev. D 88 (2013) 056011, arXiv: 1305.7099 [hep-ph]; Phys. Lett. B 735 (2014) 12, arXiv:1404.0293 [hep-ph]; EPJ Web Conf. 80 (2014) 00043, arXiv:1407.5512 [hep-ph].

[5] W. Lucha, D. Melikhov, and S. Simula, EPJ Web Conf. 80 (2014) 00046, arXiv:1410.6684 [hepph]; arXiv:1411.3890 [hep-ph]; AIP Conf. Proc. 1701 (2016) 050007, arXiv:1411.7844 [hepph]; Phys. Rev. D 91 (2015) 116009, arXiv:1504.03017 [hep-ph]; PoS (EPS-HEP2015) 532, arXiv:1508.07595 [hep-ph].

[6] B. Colquhoun et al., HPQCD Coll., Phys. Rev. D 91 (2015) 114509, arXiv:1503.05762 [hep-lat].

[7] K. G. Chetyrkin and M. Steinhauser, Phys. Lett. B 502 (2001) 104, arXiv:hep-ph/0012002; Eur. Phys. J. C 21 (2001) 319, arXiv:hep-ph/0108017.

[8] M. Jamin and B. O. Lange, Phys. Rev. D 65 (2002) 056005, arXiv:hep-ph/0108135.

[9] P. Gelhausen, A. Khodjamirian, A. A. Pivovarov, and D. Rosenthal, Phys. Rev. D 88 (2013) 014015, arXiv:1305.5432 [hep-ph]; 89 (2014) 099901(E); 91 (2015) 099901(E).

[10] K. A. Olive et al., Particle Data Group, Chin. Phys. C 38 (2014) 090001.

[11] S. Aoki et al., FLAG Working Group, Eur. Phys. J. C 74 (2014) 2890, arXiv:1310.8555 [hep-lat].

[12] W. Lucha, D. Melikhov, and S. Simula, HEPHY-PUB 973/16 (2016), in preparation.

[13] G. M. de Divitiis et al., RM123 Coll., J. High Energy Phys. 04 (2012) 124, arXiv:1110.6294 [hep-lat]; R. J. Dowdall et al., HPQCD Coll., Phys. Rev. Lett. 110 (2013) 222003, arXiv:1302. 2644 [hep-lat]; A. Bazavov et al., Fermilab Lattice and MILC Collaborations, Phys. Rev. D 90 (2014) 074509, arXiv:1407.3772 [hep-lat]; N. H. Christ et al., RBC and UKQCD Collaborations, Phys. Rev. D 91 (2015) 054502, arXiv:1404.4670 [hep-lat]. 